\section{A priori odds}

R.-D. Hilgers ${ }^{1}$, N. Heussen ${ }^{1}$ und S. Stanzel ${ }^{2}$

${ }^{1}$ Institut für Medizinische Statistik, Universitätsklinikum der RWTH Aachen, Aachen, Deutschland

${ }^{2}$ Heidelberg, Deutschland

Synonym(e) Vortest odds

Englischer Begriff prior odds; a priori odds

Definition Das a priori odds gibt das Verhältnis der Wahrscheinlichkeit dafür an, dass eine zufällig ausgewählte Person erkrankt ist, zur Wahrscheinlichkeit dafür, dass eine zufällig ausgewählte Person nicht erkrankt ist.
Beschreibung Der Begriff wird häufig im Zusammenhang mit diagnostischen Tests ( $\triangleright$ Test, diagnostischer) verwendet. Das a priori odds wird üblicherweise geschätzt durch den Quotienten

$$
\frac{\frac{\mathrm{a}+\mathrm{c}}{\mathrm{n}}}{1-\frac{\mathrm{a}+\mathrm{c}}{\mathrm{n}}}
$$

(Bezeichnungen s. Tabelle im Stichwort $>$ Vierfeldertafel).

\section{Literatur}

Zweig MH, Campbell G (1993) Receiver-operating characteristic (ROC) plots: a fundamental evaluation tool in clinical medicine. Clin Chem 39:561-577 